\title{
LCE: an open web portal to explore gene expression and clinical associations in lung cancer
}

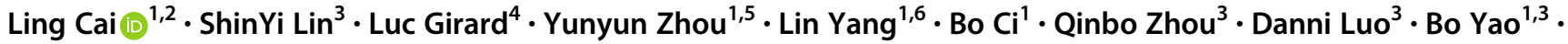 \\ Hao Tang ${ }^{1} \cdot$ Jeffrey Allen ${ }^{1} \cdot$ Kenneth Huffman $^{4} \cdot$ Adi Gazdar $^{4,7} \cdot$ John Heymach $^{8} \cdot$ Ignacio Wistuba $^{9}$. \\ Guanghua Xiao ${ }^{1,3,10} \cdot$ John Minna $a^{4,10,11,12} \cdot$ Yang Xie ${ }^{1,3,10}$
}

Received: 26 June 2018 / Revised: 4 September 2018 / Accepted: 5 September 2018 / Published online: 7 December 2018

(c) The Author(s) 2018. This article is published with open access

\begin{abstract}
We constructed a lung cancer-specific database housing expression data and clinical data from over 6700 patients in 56 studies. Expression data from 23 genome-wide platforms were carefully processed and quality controlled, whereas clinical data were standardized and rigorously curated. Empowered by this lung cancer database, we created an open access web resource- the Lung Cancer Explorer (LCE), which enables researchers and clinicians to explore these data and perform analyses. Users can perform meta-analyses on LCE to gain a quick overview of the results on tumor vs non-malignant tissue (normal) differential gene expression and expression-survival association. Individual dataset-based survival analysis, comparative analysis, and correlation analysis are also provided with flexible options to allow for customized analyses from the user.
\end{abstract}

Supplementary material The online version of this article (https:// doi.org/10.1038/s41388-018-0588-2) contains supplementary material, which is available to authorized users.

\footnotetext{
$\triangle$ Guanghua Xiao

Guanghua.Xiao@UTSouthwestern.edu

$\triangle$ John Minna

John.Minna@UTSouthwestern.edu

$\triangle$ Yang Xie

Yang.Xie@UTSouthwestern.edu

1 Quantitative Biomedical Research Center, Department of Clinical Sciences, UT Southwestern Medical Center, 5323 Harry Hines Blvd, Dallas, TX 75390, USA

2 Children's Medical Center Research Institute, UT Southwestern Medical Center, 5323 Harry Hines Blvd, Dallas, TX 75390, USA

3 Bioinformatics Core Facility, UT Southwestern Medical Center, 5323 Harry Hines Blvd, Dallas, TX 75390, USA

4 Hamon Center for Therapeutic Oncology Research, University of Texas Southwestern Medical Center, 5323 Harry Hines Blvd, Dallas, TX 75390, USA

5 Department of Data Science, University of Mississippi Medical
}

\section{Introduction}

Lung cancer is the leading cause of cancer-related death worldwide. Despite tremendous efforts put toward diagnosis and treatment, the five-year survival rate of lung cancer is still as low as $18 \%$ [1]. Over the past few decades, advancements in genome profiling techniques have greatly improved our understanding of cancer development at the

Center, 2500N State St, Jackson, MS 39216, USA

6 Department of Pathology, National Cancer Center/Cancer Hospital, Chinese Academy of Medical Sciences and Peking Union Medical College, Beijing 100021, China

7 Department of Pathology, University of Texas Southwestern Medical Center, 5323 Harry Hines Blvd, Dallas, TX 75390, USA

8 Department of Thoracic/Head and Neck Medical Oncology, University of Texas MD Anderson Cancer Center, Houston, TX 77005, USA

9 Department of Translational Molecular Pathology, University of Texas MD Anderson Cancer Center, Houston, TX 77030, USA

10 Simmons Cancer Center, University of Texas Southwestern Medical Center, 5323 Harry Hines Blvd, Dallas, TX 75390, USA

11 Department of Pharmacology, University of Texas Southwestern Medical Center, 5323 Harry Hines Blvd, Dallas, TX 75390, USA

12 Department of Internal Medicine, University of Texas Southwestern Medical Center, 5323 Harry Hines Blvd, Dallas, TX 75390, USA 

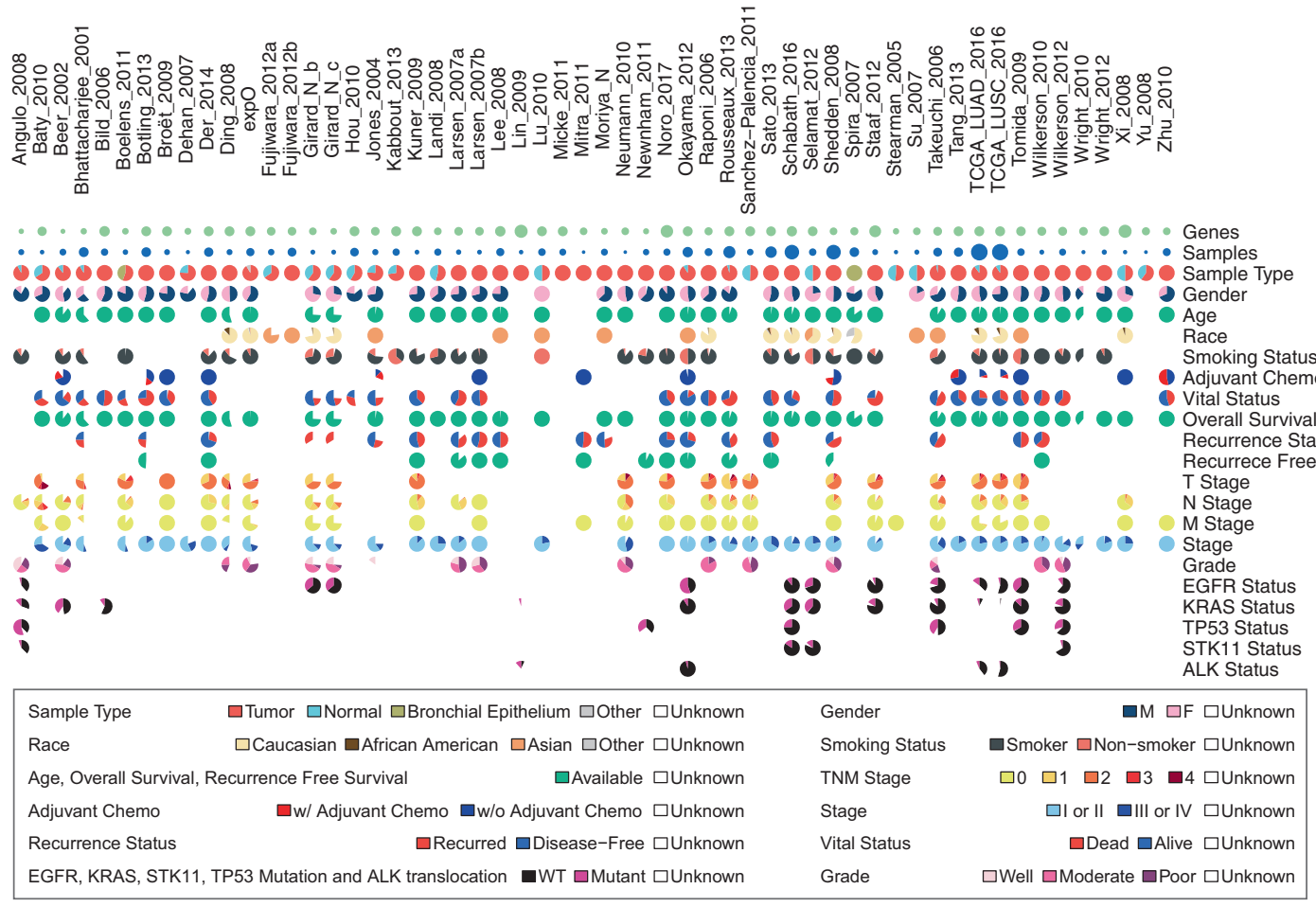

Fig. 1 Summary of lung cancer database variable distribution. This summary describes the datasets and features of the lung cancer database that feeds into the Lung Cancer Explorer. Gene expression data and clinical data were collected from 56 studies that include over 6700 patients. For each study and each variable, a pie chart is used to summarize the data. The color scheme for the pie chart sectors are provided below the gridded pie charts. Table S2 provides the specific sample sizes under each category molecular level, and have enabled the discovery of biomarkers that facilitate individualized cancer treatments including lung cancer [2]. Recent advances in immuneoncology of lung cancer also show the great importance of marker expression, the tumor mutation burden, and determination of the tumor microenvironment from deposited molecular analyses of lung cancer datasets [3-7]. With the advent of public data repositories of genome profiling data, such as the Gene Expression Omnibus (GEO, [8]), ArrayExpress [9, 10], and The Cancer Genomics Atlas (TCGA), it has become increasingly important and beneficial for researchers to mine the available datasets to discover potential biomarkers and test new biological hypotheses.

Despite the wealth of information offered by such data, utilization of public datasets is not easy, and often it can be prohibitively challenging. There is a plethora of lung cancer patient data published each year, but the data are scattered around in different public data depositories or at individual websites. There are often inconsistencies for the same patient cohort among different websites, likely due to differences in preprocessing approaches and the versions of platform annotations. Moreover, clinical records from different studies are often summarized using different terminologies. Proper usage of publicly available datasets requires specialized expertize in acquiring, processing, normalizing, and filtering of the data, which is challenging for general researchers and clinicians. To facilitate researchers' use of public datasets for biomarker discovery, a number of re-annotated database have been developed, including OncoMine [11], GeneSapien [12], Gemma [13], M2DB [14], CancerMA [15], cBioPortal [16], KMPlot [17], PrognoScan [18], PROGgene [19] and so forth.

In this study, we describe our development of a new data commons, Lung Cancer Explorer (LCE) with a web application (http://lce.biohpc.swmed.edu/), populated by a centralized lung cancer database. Compared to other existing databases, our database houses the largest collection of lung tumor expression data from 56 studies for over 6700 patients enriched with rigorously curated clinical data (Fig. 1, Tables S1 and S2). Of special note, tremendous effort was made in manual curation and standardization of the datasets so that they could be used for meta-analysis. This "harmonization" is an important benefit of LCE. Equally important, the user-friendly open web portal provides several easy but versatile analysis tools. These tools include meta-analysis, which enables users to gain a quick overview of the results from all datasets while combining statistical power from multiple datasets, as well as individual dataset-based analyses that allow for more flexibility and customization from the user. 


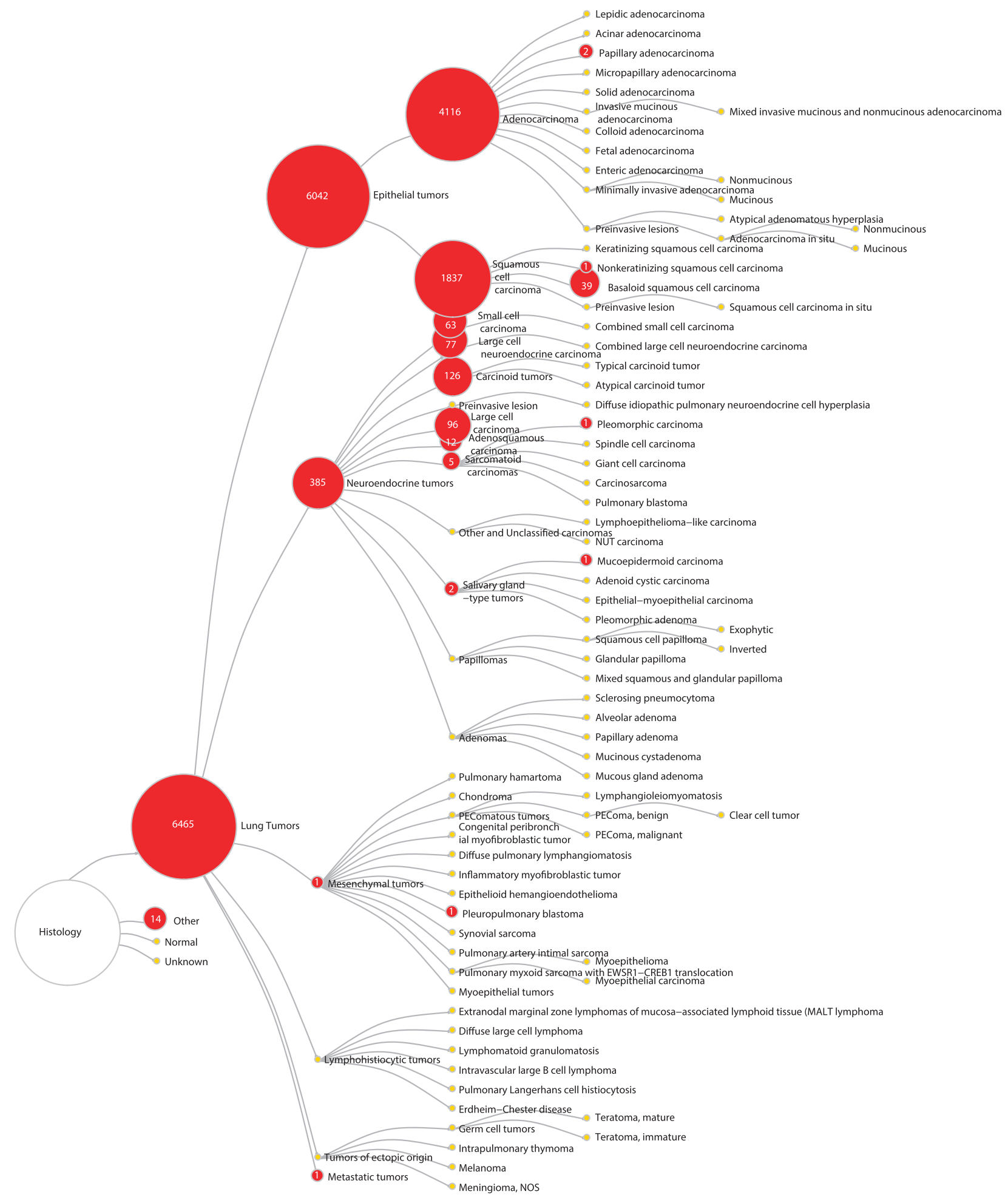

Fig. 2 Histology classification of samples collected in the lung cancer database. This tree diagram represents the hierarchical structure of the 2015 WHO classification system of lung tumors. Numbers on the red nodes denote the number of samples from the lung cancer database belonging to the corresponding histology type 

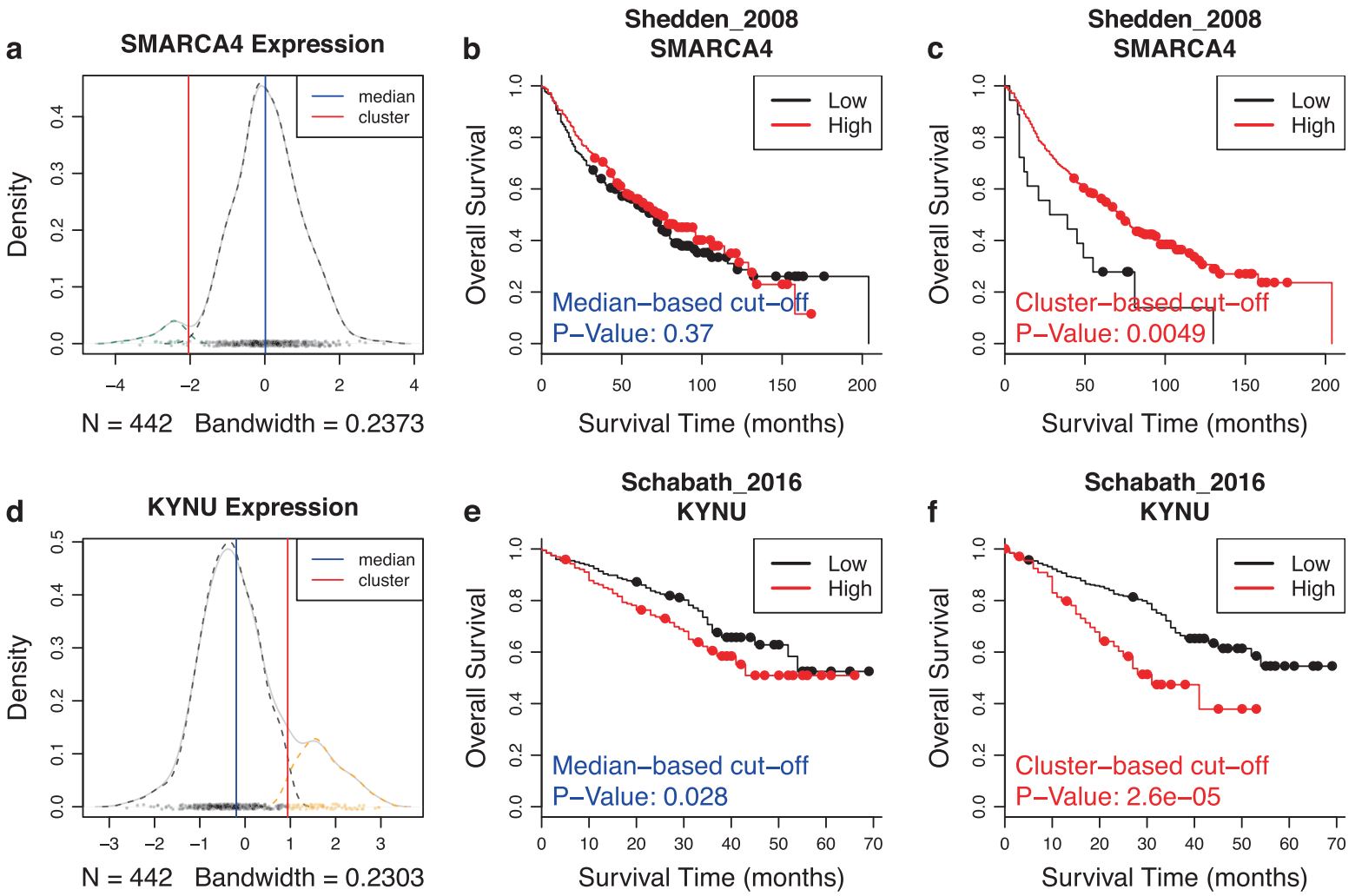

Fig. 3 Examples of survival analysis with more significant results when cluster-based cutoff is used. a Bi-modal distribution of expression in Shedden_2008 dataset. The solid blue line marks the cutoff at the median, whereas the solid red line marks the cutoff determined by Gaussian mixture model. b Kaplan-Meier curves from the survival analysis of Shedden_2008 using groups defined by SMARCA4 gene expression with cutoff at median. $P$-value from the log-rank test is

\section{Results}

\section{Construction of the lung cancer database}

Over a span of 5 years, we have collected 56 datasets generated by 23 genome-wide expression platforms (see "Data collection", "Clinical data curation", and "Expression data processing" sections in Supplementary Methods). The overarching goal is to include datasets with large numbers of samples, as well as datasets with more comprehensive coverage of clinical information with an emphasis on survival data. The number of samples in the studies we have collected has a median of 100 , maximum of 576 , and minimum of 27.

The availability and distribution of clinical variables across all studies are summarized in Fig. 1 and Table S2. The clinical variables we collected include tumor histology as defined by the 2015 WHO lung tumor classification system (Fig. 2), as well as patient demographics, diagnosis, adjuvant therapy status, smoking status, recurrence-free denoted at the bottom left corner of the plot. c Survival analysis of Shedden_2008 using groups defined by Gaussian mixture model of SMARCA4 expression. d Bi-modal distribution of $K Y N U$ expression in Schabath_2016 dataset. e Survival analysis of Schabath_2016 using groups defined by SMARCA4 gene expression with cutoff at median. $\mathbf{f}$ Survival analysis of Schabath_2016 using groups defined by Gaussian mixture model of $K Y N U$ expression

and overall survival time and status, and mutation status of some key cancer genes (Fig. 1 and Table S2). Extensive quality control measures were taken for assessment of the expression data and clinical data. Details of these measures are described in the Supplementary Methods (also see Figure S3).

\section{Lung Cancer Explorer}

Having established a high-quality lung cancer database, we constructed the user-friendly website LCE (http://lce. biohpc.swmed.edu), allowing the cancer research community to gain easy access to our resources. Our dataset inventory and sources are described on the DATA page of LCE. Processed data are available for user download under each study. The ANALYSIS page of LCE provides survival analysis, comparative analysis and co-expression analysis tools based on individual datasets, as well as meta-analysis tools based on multiple datasets. The functionality of these tools is described in detail in the following sections. 


\section{a RORCTumor vs Normal Meta-analysis Adenocarcinoma}

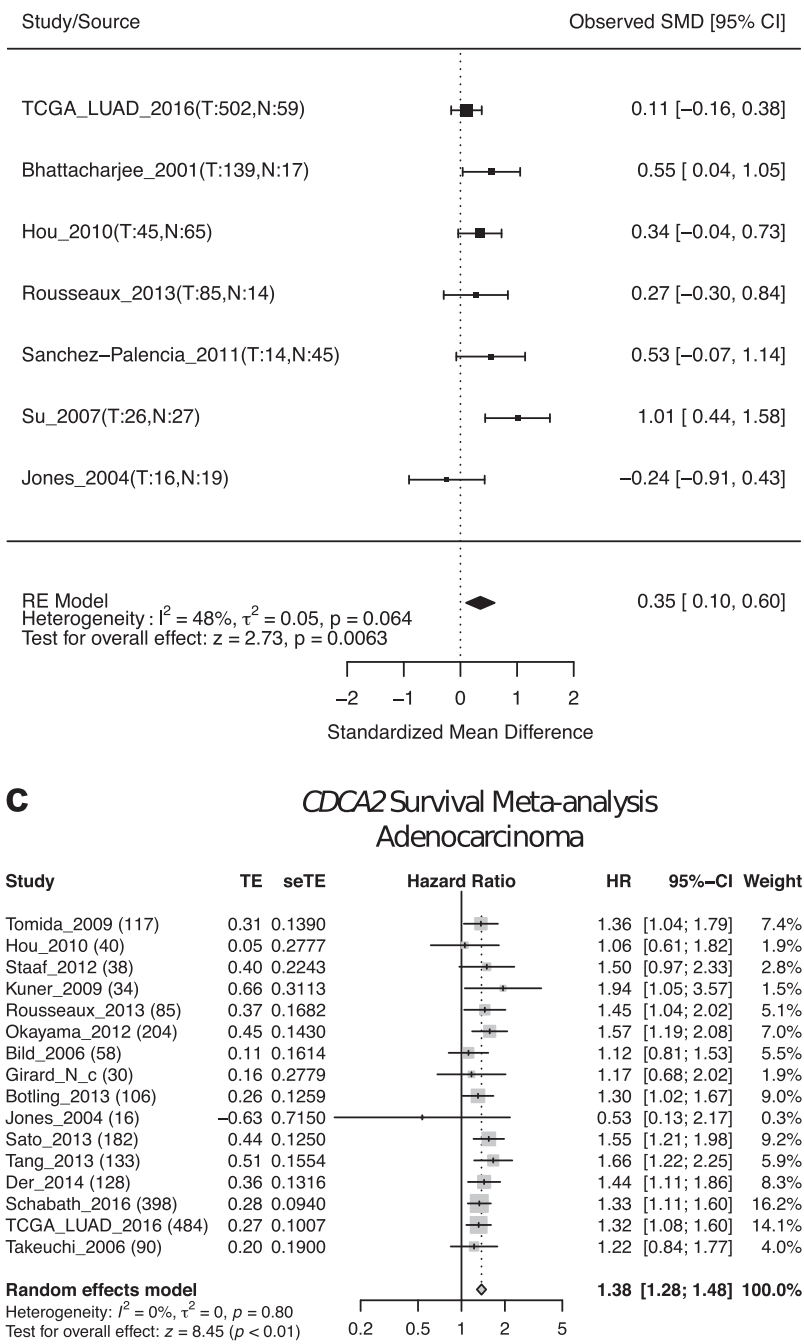

Fig. 4 High klotho expression has more significant association with positive survival outcome in males. For each of the six selected studies, survival analysis assessing prognosis association of KL gene expression was performed for male patients or female patients only. In

\section{Survival analysis in LCE}

\section{Flexible group dichotomization}

Survival analysis is commonly provided in online cancer databases to allow users to assess the association between gene expression and prognosis, and the median is routinely used as the dichotomization cutoff for the continuous gene expression. However, gene expression pattern is often a result of heterogeneous oncogenotypes and the distribution is often unbalanced. The LCE survival analysis module offers four options for cutoff value, including "median", "mean", "cluster", and "custom". In the Results panel, a Kaplan-Meier plot, table of summary statistics and Kernel density plot of the expression data are provided to the user. b RORCTumor vs Nomal Meta-analysis Squamous Cell Carcinoma

\begin{tabular}{lll} 
Study/Source & Observed SMD [95\% Cl] \\
\hline TCGA_LUSC_2016(T:468,N:51) & $\vdots$ & $-2.07[-2.39,-1.76]$ \\
Hou_2010(T:27,N:65) & $-2.44[-3.01,-1.87]$ \\
Fujiwara_2012a(T:49,N:30) & $-2.20[-2.77,-1.63]$ \\
Sanchez-Palencia_2011(T:31,N:45) & & $-2.32[-2.91,-1.74]$ \\
Rousseaux_2013(T:61,N:14) & & $-3.10[-3.86,-2.33]$ \\
Bhattacharjee_2001(T:21,N:17) & & $-1.76[-2.65,-0.86]$
\end{tabular}

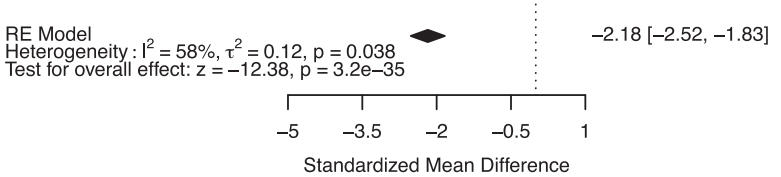

d

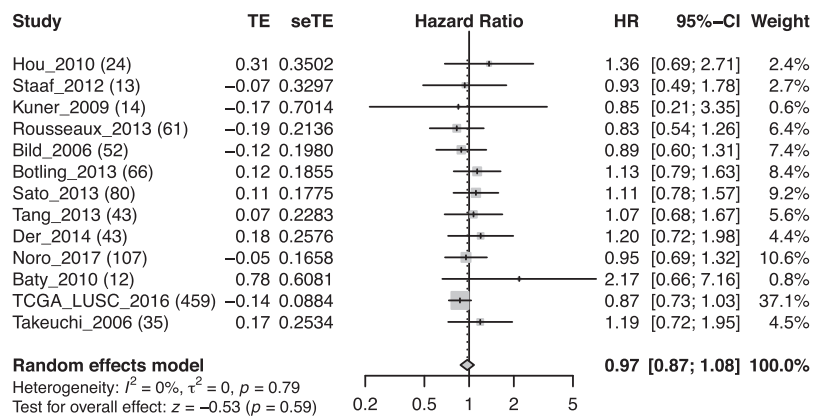

each analysis, the median was used as a cutoff for dichotomizing patients. In all six studies, a more significant association with better prognosis was found in the male patients compared to the female patients

The density plot visualizes the distribution of the gene expression and facilitates the user in determining whether they should modify their choice of cutoff. In particular, the "cluster" option in cutoff selection would be a more rational choice for bimodally distributed expression values, as it separates the sample groups by a cutoff estimated from Gaussian mixture modeling.

\section{Survival analysis examples with cluster-based cutoff}

In Fig. 3 we provide examples using the genes SMARCA4 and $K Y N U$ in two lung adenocarcinoma (ADC) studies. Bimodal distribution of gene expression was observed in both cases (Fig. 3a, d). SMARCA4, a well-known tumor suppressor gene [20] that can serve as prognostic indicators in 


\section{a RORCTumor vs Normal Meta-analysis Adenocarcinoma}

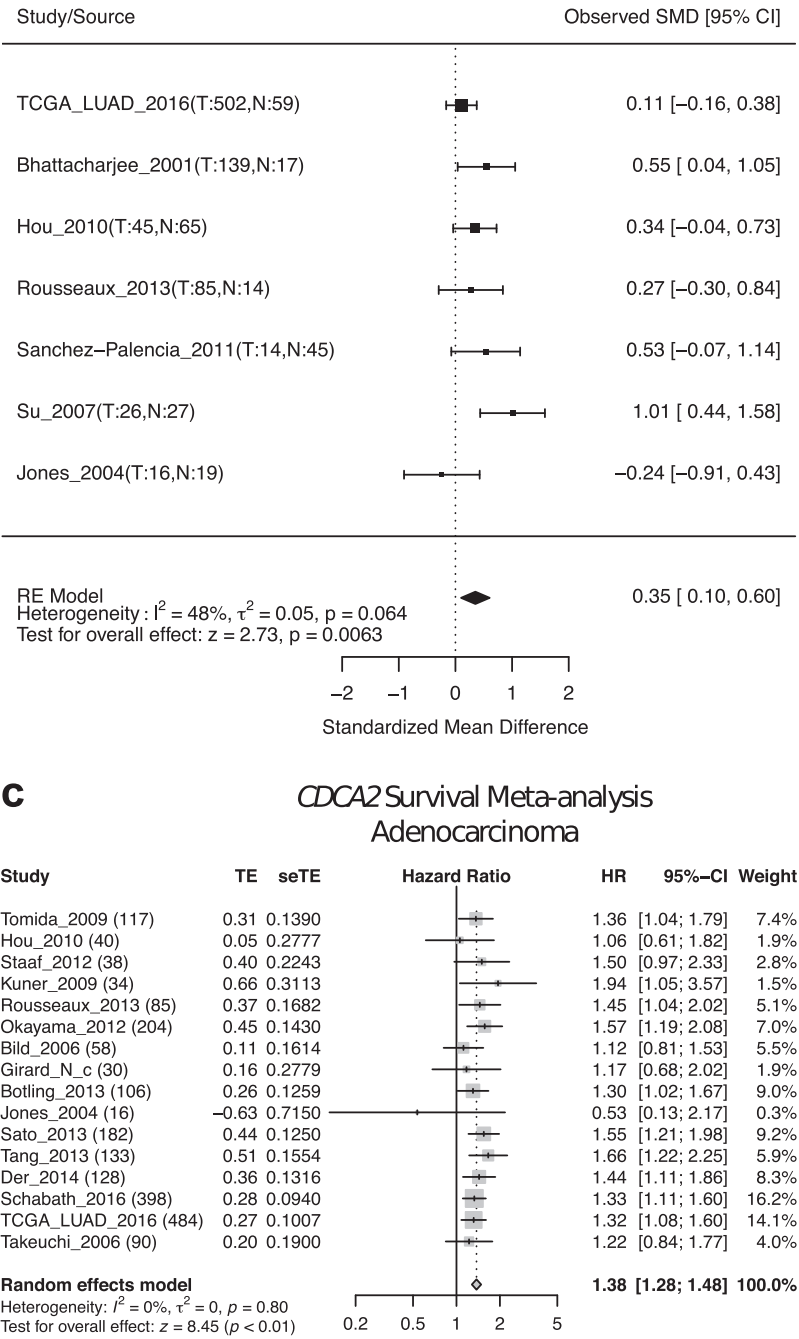

Fig. 5 Examples of different meta-analysis results in lung adenocarcinoma vs squamous cell carcinoma. a, b RORC tumor vs normal meta-analyses in lung ADC studies (a) and lung SCC studies (b). c, d $C D C A 2$ survival meta-analyses in lung ADC studies (a) and lung SCC studies. Note that differential gene expression meta-analysis for RORC is only significant in lung SCC patients, whereas survival meta-

non-small cell lung cancer [21] and breast cancer [22], was under-expressed in a small fraction of samples from the Shedden_2008 study [23], and the corresponding patients had worse survival outcome (Fig. 3c). In contrast, KYNU was over-expressed in a small proportion of samples in dataset Schabath_2016 [24] and the corresponding patients also had worse survival outcome. In both cases, results from survival analysis were more significant when the cutoff was selected by "cluster" as opposed to "median" (Fig. 3b, c, e, f). With the built-in "cluster" option for cutoff selection, users can easily generate figures like Fig. 3c, f and compare them with the default "median" options like Fig. 3b, e. b RORCTumor vs Nomal Meta-analysis Squamous Cell Carcinoma

\begin{tabular}{lll} 
Study/Source & Observed SMD [95\% Cl] \\
\hline TCGA_LUSC_2016(T:468,N:51) & $\vdots$ & $-2.07[-2.39,-1.76]$ \\
Hou_2010(T:27,N:65) & & $-2.44[-3.01,-1.87]$ \\
Fujiwara_2012a(T:49,N:30) & $-2.20[-2.77,-1.63]$ \\
Sanchez-Palencia_2011(T:31,N:45) & & $-2.32[-2.91,-1.74]$ \\
Rousseaux_2013(T:61,N:14) & & $-3.10[-3.86,-2.33]$ \\
Bhattacharjee_2001(T:21,N:17) & & $-1.33[-2.04,-0.63]$ \\
Baty_2010(T:12,N:15) & & $-1.76[-2.65,-0.86]$
\end{tabular}

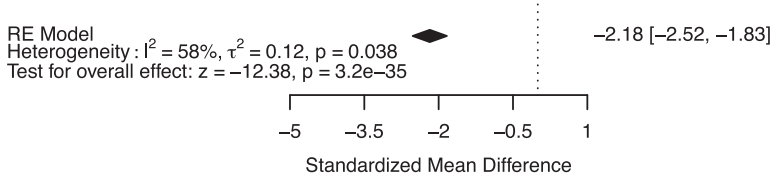

d

CDCA2Sunvival Meta-analysis Squamous Cell Carcinoma

$\begin{array}{llll}\text { Study } & \text { TE seTE Hazard Ratio HR } 95 \%-\mathrm{Cl} \text { Weight }\end{array}$

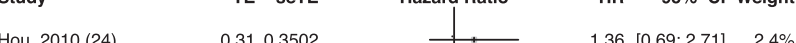
Hou_2010(24) $\quad 0.310 .3502$ Staaf_2012 (13) $\quad-0.070 .3297$ Kuner_2009 (14) $\quad-0.170 .7014$ Rousseaux_2013 (61) $\quad-0.190 .2136$ Bild_2006 (52) $\quad-0.120 .1980$ $\begin{array}{ll}\text { Botling_2013(66) } & 0.120 .1855 \\ \text { Sato_2013 (80) } & 0.110 .1775\end{array}$ Sato_2013(80) $\quad 0.110 .1775$ Tang_2013 (43) $\quad 0.070 .2283$ $\begin{array}{lr}\text { Der_2014 (43) } & 0.180 .2576 \\ \text { Noro_2017 (107) } & -0.050 .1658\end{array}$ Noro_2017 (107) $\quad-0.050 .1658$ Baty_2010(12) 0.780 .608 TCGA_LUSC_2016 (459) -0.140 .0884$

Takeuchi_2006(35) 0.170 .2534

Random effects model

Heterogeneity: $I^{2}=0 \%, \tau^{2}=0, p=0.79$
Test for overall effect: $z=-0.53(p=0.59)$

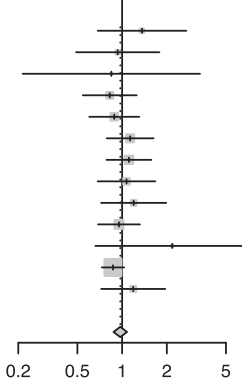

$0.93[0.49 ; 1.78] \quad 2.7 \%$ $0.85[0.21 ; 3.35] \quad 0.6 \%$ $0.83[0.54 ; 1.26] \quad 6.4 \%$ $\begin{array}{ll}0.89[0.60 ; 1.31] & 7.4 \% \\ 1.13[0.79 \cdot 1.63] & 8.4 \%\end{array}$ $\begin{array}{ll}1.13[0.79 ; 1.63] & 8.4 \% \\ 1.11[0.78 ; 1.57] & 9.2 \%\end{array}$ $\begin{array}{ll}1.11[0.78 ; 1.57] & 9.2 \% \\ 1.07[0.68 ; 1.67] & 5.6 \%\end{array}$ $1.20[0.72 ; 1.98] \quad 4.4 \%$ $1.20[0.72 ; 1.98]$
$0.95[0.69 ; 1.32]$
$10.6 \%$ $\begin{array}{rr}0.95[0.69 ; 1.32] & 10.6 \% \\ 2.17[0.66 ; 7.16] & 0.8 \%\end{array}$ $0.87[0.73 ; 1.03] \quad 37.1 \%$ $1.19[0.72 ; 1.95] \quad 4.5 \%$

$0.97[0.87 ; 1.08] 100.0 \%$

analysis for $C D C A 2$ is only significant in lung ADC patients. In each forest plot, the name of each study is followed by the number of tumor and normal samples (tumor vs normal meta-analysis) or total tumor samples (survival meta-analysis). SMD standardized mean difference, TE estimated treatment effect, seTE standard error of treatment effect, HR hazard ratio, CI confidence interval

\section{Analysis stratification by additional clinical variables}

In the LCE survival analysis module, options are provided for users to select a group of patients by age, race, gender, smoking status, and histology. This allows users to assess the association between the expression of a user-selected gene and patient survival (gene-survival association) within a user-defined subpopulation of patients. An example in Fig. 4 is provided to illustrate the advantage of this approach in the identification of a gender-specific genesurvival association. In Fig. 4, association of KL gene expression and survival was tested separately in female and in male patients. We show that for several studies, a 
stronger positive association between high klotho gene expression and overall survival could be observed in male patients as compared to female patients. Klotho, encoded by gene $K L$, is a well characterized anti-aging gene [25]. It has been observed that the extension of lifespan by klotho overexpression is more pronounced in males than in females [26], and only male but not female klotho mutant mice responded to a phosphorus restriction diet to extend lifespan [27]. In recent years, klotho has also been characterized as a tumor suppressor gene [28]. From our analyses, it is interesting to see that the tumor suppressing effect of klotho also seems to be higher in males than in females (Fig. 4).

\section{Meta-analysis in LCE}

\section{Types of meta-analysis}

In LCE, meta-analysis tools are provided to allow users to address two questions: (1) differential expression between tumor and normal samples; and (2) survival association of gene expression.

\section{Cohort-specific meta-analysis and examples}

Results from both types of meta-analyses are visualized as forest plots. We provide three options, "All Cancers", "Adenocarcinoma" (ADC), or "Squamous Cell Carcinoma" (SCC), to allow users to choose the lung cancer subtype(s) they want to include in the meta-analysis since the survival association and expression difference between tumor and normal could be cancer-type specific.

For example, with lung cancer subtype-specific metaanalysis, we found consistent downregulation of RAR related orphan receptor $\mathrm{C}(\mathrm{RORC})$ in multiple lung $\mathrm{SCC}$ studies (Fig. 5b) but not in lung ADC studies (Fig. 5a). Interestingly, $R O R C$ was also previously found in a 3-gene signature to distinguish lung ADC and lung SCC [29]. We also found that in multiple lung ADC studies, expression of cell division cycle-associated protein 2 (CDCA2) was associated with worse overall survival outcome (Fig. 5c), whereas this trend was not observed for lung SCC datasets (Fig. 5d).

\section{Validation of tumor versus normal gene expression difference meta-analysis}

With access to qPCR measurements of 46 nuclear hormone receptor genes in 30 pairs of matched tumor and normal lung cancer samples, we were able to compare the standardized mean difference between tumor and normal tissue gene expression estimated from meta-analysis to the qPCR measurement results. A strong agreement was observed
Standardized Mean Difference Tumor - Normal of 48 Nuclear Hormone Receptor Genes

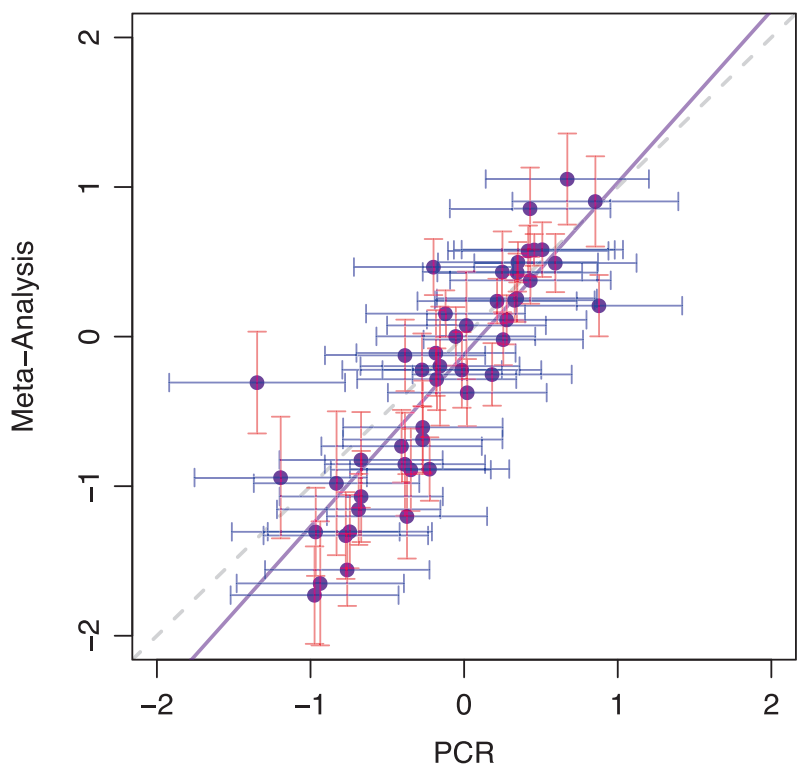

Fig. 6 Meta-analysis estimates agree with qPCR measurements on tumor vs normal expression differences for 46 nuclear hormone receptor genes. Results from qPCR measurements of 30 tumor-normal pairs ( $x$-axis values) and meta-analysis estimates from 21 studies $(y$ axis values) on gene expression differences between tumor and normal tissues for 46 nuclear hormone receptor genes were used to evaluate consistency between the two approaches. The values on the $x$-axis and $y$-axis are the standardized mean difference estimated by Hedges' G method. The solid purple line represents a linear regression line, whereas the dashed gray line identifies where $x$ equals $y$

between the two results, supporting the validity of our metaanalysis and the high quality of our datasets (Fig. 6, Table S5).

\section{Assessing reproducibility across different studies from meta-analysis}

Meta-analysis is a unique tool provided by LCE, as it not only provides users with statistical estimates that are more precise than using any single dataset, it also allows users to recognize the extent of reproducibility of a specific analysis across different datasets. In the forest plots generated by the LCE meta-analysis module, we provide users with a heterogeneity test using the $\mathrm{I}^{2}$ statistic, which describes the percentage of variation across studies that is due to heterogeneity [30]. It is important to note that inconsistency in the results between different studies could arise from differences in patient population or sample procurement, as well as in data acquisition. In some cases, the results are more consistent for specific genes than others (Fig. 7). Hence, the meta-analysis tool provided by LCE allows users to identify discrepancies among different datasets in order to estimate the generalizability of the results. 
a

Distribution of $\mathrm{I}^{2}$ from adenocarcinoma-specific survival meta-analysis

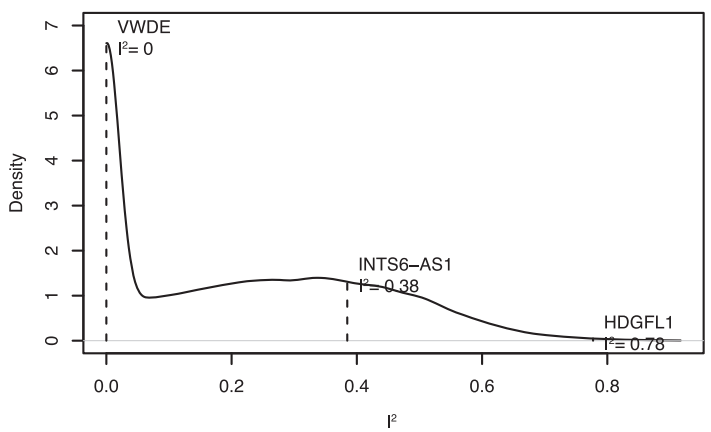

b

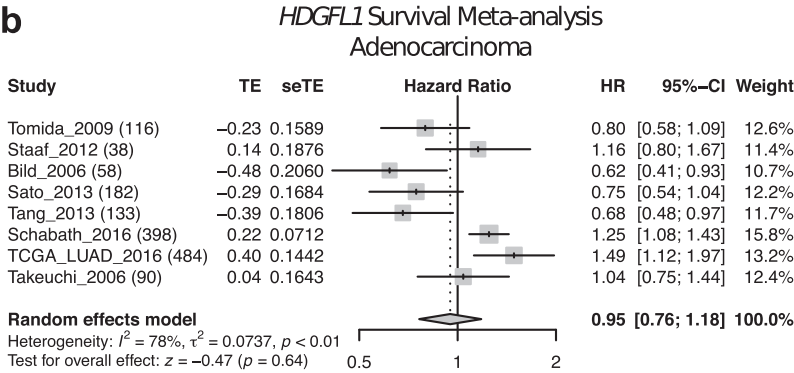

C

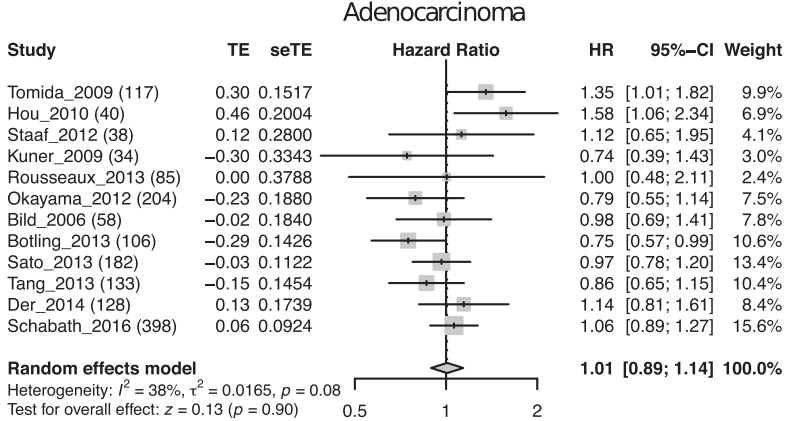

d WWDESunvival Meta-analysis

\begin{tabular}{|c|c|c|c|c|c|}
\hline & & nocarcinom & & & \\
\hline Study & TE SeTE & Hazard Ratio & HR & $95 \%-\mathrm{Cl}$ & Weight \\
\hline Tomida_2009 (117) & 0.310 .1426 & $\because$ & 1.36 & {$[1.03 ; 1.80]$} & \\
\hline Hou_2010 (40) & 0.280 .22 & & 1.32 & {$[0.85 ; 2.06]$} & \\
\hline & $0.18 \quad 0.2$ & 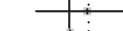 & 1.20 & & $2.1 \%$ \\
\hline Kuner $2009(34)$ & & $\therefore$ & 1.01 & {$[0.59 ; 1.71]$} & $2.0 \%$ \\
\hline usseaux_2013 (85) & $0.44 \quad 0.1$ & $\div$ & 1.56 & & $4.3 \%$ \\
\hline Okayama_2012 (204) & 0.290 .1 & $\therefore$ & 1.34 & {$[1.03 ; 1.75]$} & $7.9 \%$ \\
\hline Bild_2006 (58) & $\begin{array}{lll}0.05 & 0.1\end{array}$ & $\because$ & 1.05 & {$[0.73 ; 1.52]$} & $4.1 \%$ \\
\hline $\begin{array}{l}\text { Girard__NC C (30) } \\
\text { Botling_213 (106) }\end{array}$ & $\begin{array}{l}0.680 .2890 \\
0.22 \\
0.1059\end{array}$ & $\doteqdot$ & $\begin{array}{l}1.96 \\
1.24\end{array}$ & $\begin{array}{l}1[1.12 ; 3.46] \\
{[1.01 ; .53]}\end{array}$ & $\begin{array}{r}1.7 \% \\
12.8 \%\end{array}$ \\
\hline Salo & 0.120 .1 & $\div$ & 1.13 & {$[0.88 ; 1.45]$} & $8.7 \%$ \\
\hline & 0.0 & $\therefore$ & & {$[0.79 ; 1.52]$} & $5.1 \%$ \\
\hline & 0.3 & $\div$ & 1.40 & {$[1.06 ; 1.84]$} & $7.4 \%$ \\
\hline Scha & 0.08 & $\div$ & 1.08 & {$[0.91 ; 1.29]$} & $17.6 \%$ \\
\hline TCGA_LUAD_2016 ( & 0.130 .0934 & 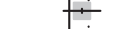 & 1.13 & {$[0.94 ; 1.36]$} & $16.4 \%$ \\
\hline & & $\therefore$ & 1.2 & [1 & $00.0 \%$ \\
\hline tt for overall effect: $z$ & $<0.0$ & & & & \\
\hline
\end{tabular}

Fig. 7 Assessment of result consistency by $\mathrm{I}^{2}$ statistics in meta-analysis of survival-gene expression association. a Density estimation of $\mathrm{I}^{2}$ distribution. Three genes with different $\mathrm{I}^{2}$ statistics were selected as examples in (b), (c), and (d). A larger $\mathrm{I}^{2}$ value suggests a larger degree of heterogeneity across studies, whereas a smaller $I^{2}$ value is reflective of a higher degree of consistency among studies. b, c, d Example forest plots of survival meta-analysis with different heterogeneity: large (b), intermediate (c), and small (d)

\section{Comparative analysis in LCE}

Comparative analysis was implemented for users to assess the associations between a user-selected gene and clinical factors such as gender, age, histology types, disease stages, etc., within a specific dataset. The expression levels of the selected gene in the user-defined patient groups are shown in boxplots and $p$ values of the expression differences are reported. In addition to group assignment based on a single clinical variable, a unique functionality of LCE is that users can define patient groups based on a combination of clinical factors. This provides a great extent of flexibility in hypothesis testing to understand the interactions between different clinical variables. For example, expression comparison of the hemoglobin subunit delta encoding gene $H B D$ in the TCGA_LUAD_2016 cohort shows that tumor samples have decreased $H B D$ expression compared to normal samples (Fig. 8a), whereas samples from smokers and non-smokers have similar expression levels (Fig. 8d). However, by stratifying patient groups with two factors, both tissue type (tumor vs normal) and smoking status, we find the difference in $H B D$ levels between normal and tumor tissues is significant only in smokers but not in nonsmokers (Fig. 8b, c), and normal samples from smokers have elevated $H B D$ expression compared to normal samples from non-smokers (Fig. 8f). In contrast, no difference in $H B D$ expression was observed for tumor tissues from smokers vs non-smokers (Fig. 8b), nor do $H B D$ expression levels differ in the tumor and normal tissues of non-smokers (Fig. 8f).

The results from these comparisons suggest that $H B D$ expression is upregulated in normal lung tissue by smoking but is downregulated again when tumors form in smokers. We also observed similar trends in the other hemoglobin subunit encoding genes $H B G 1, H B G 2$, and $H B M$, which is consistent with the previous finding that hemoglobin levels increase in smokers [31].

\section{Correlation analysis in LCE}

The correlation analysis tool from LCE provides users a heatmap to visualize the expression correlations among a list of user-defined genes in user-selected datasets. A high degree of expression correlation of genes often implies functional association, as genes involved in the same pathway or biological function are often subject to concerted regulation at transcription level [32]. Functional partners of the same gene could differ in a tissue-specific manner [33], and the gene network could also re-wire under a different disease context. In LCE we provide three options, "All", "Lung Tumor" and "Normal", to allow users to calculate a gene expression correlation matrix based on a specific sample type and subsequently generate a clustered 

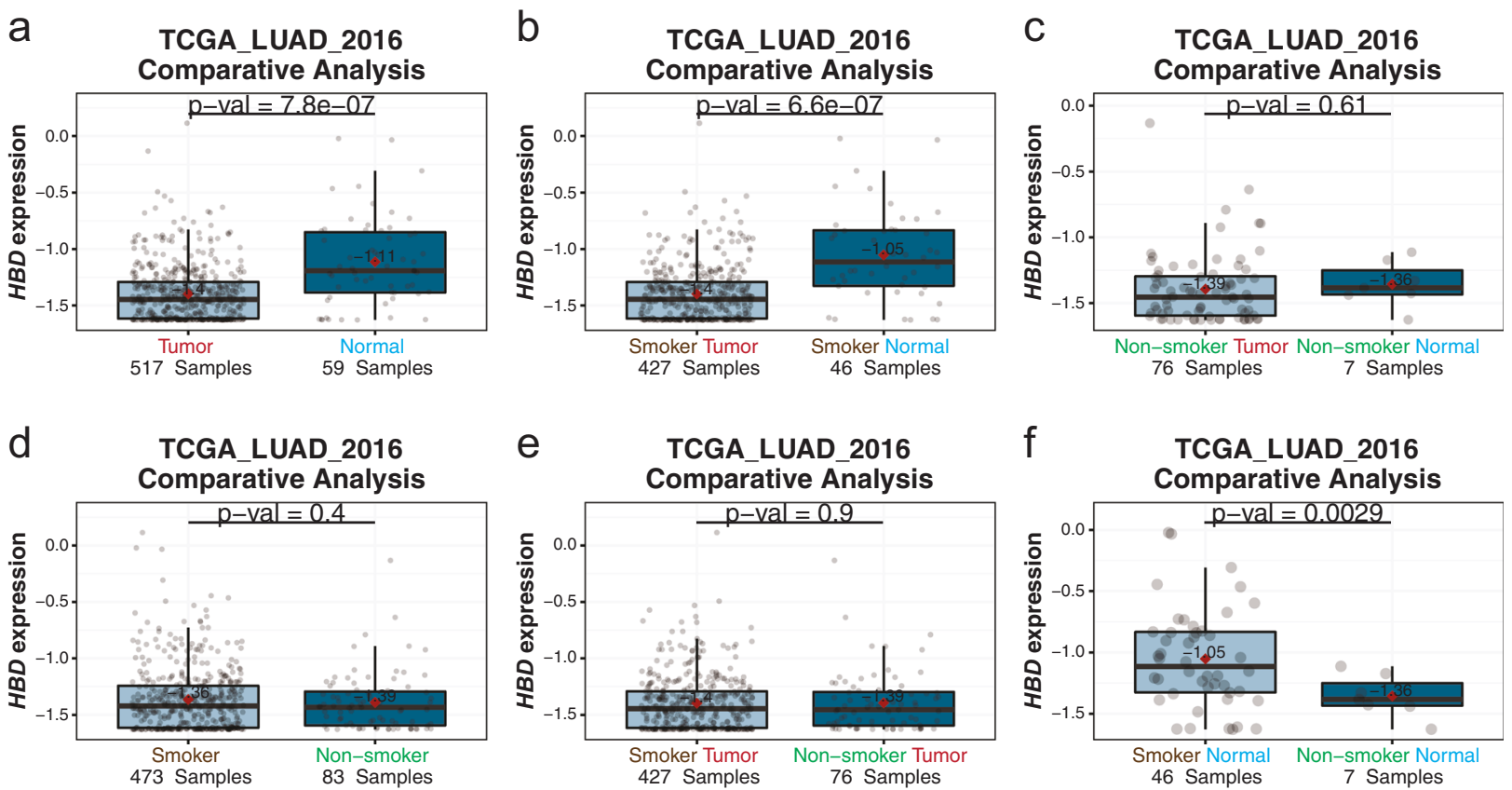

Fig. 8 Interaction between sample tissue type and smoking status in HBD gene expression. a, d Boxplots comparing $H B D$ gene expression between two groups dichotomized on a single clinical variable: tissue type (a) or smoking status (d). b, c, e, f Boxplots comparing $H B D$ gene

expression between two groups defined by a combination of two clinical variables: different tissues in smoker (b), different tissues in non-smoker (c), tumor from patients with different smoking status (e), and normal tissues from patients with different smoking status (f)

heatmap, which conveniently allows users to identify changes in the co-expression patterns of the user-defined gene list. One such example is provided in Fig. 9, where we show that in tumor, there is a high degree of co-expression between poly(ADP-ribose) polymerase-2 (PARP2) and 10 cell cycle genes (Fig. 9a) selected from MSigDB "REACTOME_CELL_CYCLE" gene set [34, 35], whereas this coexpression is diminished in normal tissues (Fig. 9b). This is consistent with the role of $P A R P 2$ in DNA repair [36]; since genomic instability and mutation is a hallmark of cancer, the cancer-specific co-expression of $P A R P 2$ and cell cycle genes may indicate that $P A R P 2$ is actively engaged in DNA repair while cancer cells divide. On the other hand, we found PARP2 highly correlated with zinc fingers $\mathrm{C} 2 \mathrm{H} 2-$ type genes (ZNF) [37] in normal but not cancer tissue (Fig. $9 \mathrm{c}, \mathrm{d})$. This normal-specific co-expression of PARP2 and ZNF genes may suggest alternative roles of $P A R P 2$ in transcriptional regulation independent of its DNA repair function.

\section{Discussion}

In this paper, we described the construction of the LCE database for lung cancer gene expression analysis. It was carefully designed for lung cancer researchers to interrogate gene expression association with patient clinical features. As the collected datasets are highly heterogeneous, extensive efforts were put forth to reprocess and normalize expression data from 23 different expression profiling platforms, and a large amount of manual curation work was performed to standardize clinical terminology. Such manual inspection, though time consuming, greatly improves the data accuracy and usability, which sets our work apart from other databases. The resulting database with high-quality datasets enables versatile analysis tools in our LCE. We provide meta-analysis tools that summarize results across multiple datasets in the form of forest plots to allow users to gain a summary view of the overall trend and heterogeneity among studies. We also provide individual dataset-based analysis tools to allow users the flexibility to intricately formulate their analysis to best fit the research question. Results and biological insights we obtained from examples (Figs. 3-5 and 7-9) demonstrated the unique advantages of our tools over the current publically available web tools, as none of these results could have been produced with the existing public tools.

We welcome users to contribute or suggest additional datasets to be evaluated and added to our lung cancer database. Suggestions can be made by leaving a comment at the contact page of LCE. It is in our plan to add a functionality to LCE to enable users to upload their own data to our database and perform analysis with our web application. In the future, we would also like to expand the lung cancer database to include cell line data and patient-derived xenograft (PDX) data. Besides gene expression data, other 
Fig. 9 Different co-expression pattern between PARP2 and cycle genes. a, b, c, d Heatmaps of gene-gene correlation matrices from

TCGA_LUAD_2016 for PARP2 and 10 selected cell cycle genes from tumor sample expression data (a) or normal sample expression data (b), and for PARP2 and 10 selected $\mathrm{C} 2 \mathrm{H} 2-$ type zinc finger genes (ZNF) from tumor sample expression data (c) or normal sample expression data (d). The highly positive correlation between $P A R P 2$ and cell cycle genes was seen only in tumor samples but not normal samples (a, b), whereas the high degree of positive correlation between PARP2 and ZNF genes was observed only in normal tissue samples but not tumor samples (c, d)

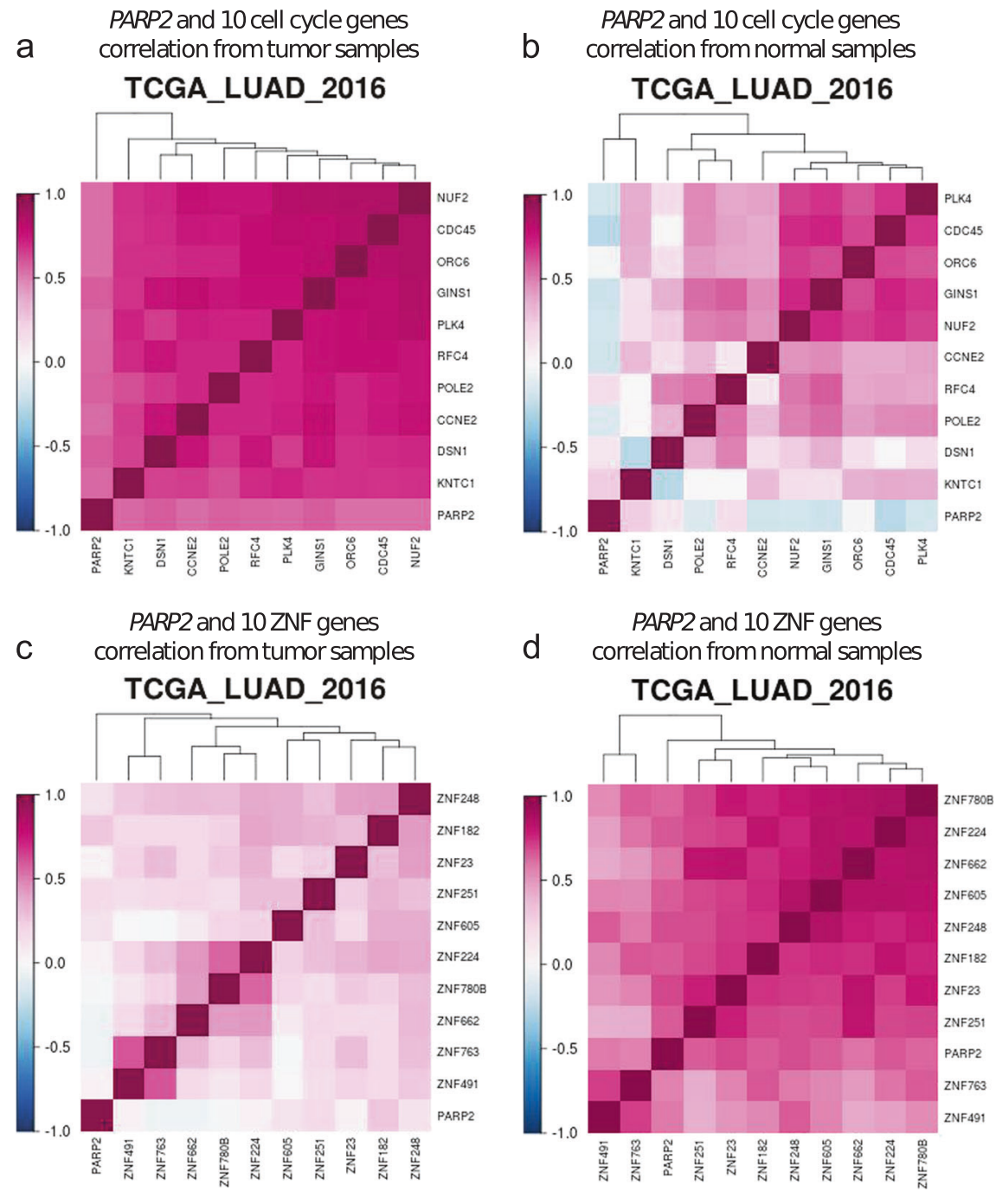

types of molecular profiling data (such as proteomic data, mutation data, copy number variation data, epigenomics data, microRNA data, etc.) and imaging data (such as H\&E pathological slide images) will also be added to the lung cancer database. Separate data tables and supporting data dictionaries will be created for the new molecular data types. We will first identify studies within our collection that possess such data and add them to our database, then look for additional datasets that contain such molecular data as well as clinical data to add to our database. We will also expand the analysis tool repertoire on LCE to include multivariate analysis and other integrative analytical approaches.

Finally, we will conduct a variety of systematic analyses with the lung cancer database to generate testable hypotheses (for example, identification of genes associated with different oncogenotypes, gender, smoking status, etc. followed by gene set enrichment analysis). Results from such systematic analyses will be provided to the lung cancer research community to provoke hypothesis generation, testing, and validation.

\section{Material and methods}

\section{Data collection and processing}

\section{Dataset selection}

Datasets were collected from GEO, TCGA, and individual literatures. The search of GEO was performed by GEOmetadb [38]. For datasets that had not been deposited into GEO, we made our selection through a literature search and by referencing other commonly used databases.

\section{Clinical data curation}

Clinical data for datasets deposited into GEO were retrieved from GEO by $\mathrm{R}$ package GEOquery; TCGA clinical data were downloaded from Sage Bionetworks' Synapse database [39], and other datasets were downloaded from sources provided in the original publication. The clinical data obtained directly from these public domains often contained non- 
standard terminology. To standardize the clinical variables from different studies, codebooks were devised for each variable in order to ensure the accuracy and compatibility of the clinical annotation from different sources (Table S4.1S4.6). The patient histology codebook was created based on the 2015 World Health Organization (WHO) Classification of Lung Tumors [40] (Fig. 2 and Table S3). In order to facilitate users in integrating our datasets with other cancer datasets, we also provided the ICDO code [40] and the corresponding SNOMED-CT code [41] for histological subtypes in the processed data included on LCE for download. For the TCGA lung cancer data in particular, instead of using the histology classification provided by the patient information file, histology was determined based on expression signature as developed by Girard et al. [42], as that study has shown improved classification accuracy with the gene expression classifier on the TCGA data. Consequently, the histology-misclassified samples were excluded from the TCGA cohorts in cancerspecific meta-analysis. For all datasets, programmatic and manual data curations were carried out and the procedures were repeated three times with scrutiny. For our records, all the data handling steps were saved with detailed documentation.

\section{Quality control of clinical data}

Manual data curation was performed to ensure consistency between supplementary information associated with the original publication and the clinical data downloaded from GEO. Clinical information found only in the original publication but not in the GEO records was also extracted. Here we describe a few examples of our manual curation from numerous instances: we checked if there were exclusion criteria in the paper that imposed restrictions on adjuvant therapy, tumor stage, etc.; when calculating the survival time we looked for surgical date, and if it was available we used it as the start date for survival time instead of the initial diagnosis date, since the gene expression data reflected the tumor profile on the surgical date; when certain samples were considered low quality and removed from analyses in the associated publication, we followed the same discretion to excl ude such samples from our collection; we removed cell line samples to ensure our collection included exclusively patient samples; when tumor percentage information was available, we removed samples with $<50 \%$ tumor content.

\section{Expression data processing}

Expression data for datasets deposited into GEO were retrieved from GEO by $\mathrm{R}$ package GEOquery. TCGA expression data were downloaded from Broad GDAD firehose [43], and other datasets were downloaded from the sources provided in the original research papers. It is not uncommon in the field of biomarker discovery for signatures to have poor reproducibility in other datasets. Such discrepancy could be at least partially attributed to the differences in experimental settings, sample handling, measurement platforms and, importantly, data processing procedures. The datasets collected in this study were generated from 23 different platforms, with the majority being microarrays. We adopted different strategies to process the data (Figure S1) to convert the expression data from probe level to gene level.

\section{Quality control of gene expression data}

To perform quality control of the expression data input for meta-analysis, a method that checks for reproducibility across studies based on the concept of the integrative correlation coefficient (ICC) [44, 45] was implemented. The premise of this approach is that most of the pairwise genegene correlation should be preserved across different studies. The relationship of reproducibility between studies could be visualized by ICC-based clustering, as shown in Figure S3. Considering that some gene-gene correlation could be tissue-type specific, samples of different tissue types from the same study were separated into distinct groups before we calculated the ICC. As expected, in clusters defined by ICC, subgroups of different sample types from the same study in many cases did not cluster together; instead, samples of the same tissue type from different studies tended to cluster together. A clade of four studies with very little correlation with other sample groups was identified. These four studies were removed from subsequent meta-analyses. However, they were still available to use in the individual dataset-based analysis. Moreover, the two RNA-seq datasets from TCGA revealed high correlation with datasets from microarray platforms, supporting the compatibility of datasets from different platforms based on our processing approach.

\section{Database structure/web interface}

Our web application LCE can be accessed through http:// lce.biohpc.swmed.edu/. It was created using PHP (7.0.12-1) in the R Programming environment (3.3.1) with MySQL database (Ver 14.14 Distrib 5.5.49) in the backend. Our MySQL database contains tables for samples, patients and gene expression data with supporting data dictionaries (Figure S2 and Table S4.1-S4.6).

\section{Code availability}

Data cleaning, processing, and analyses were performed using R. R scripts are available upon request. 


\section{Statistical analysis methods}

\section{Cluster-based cutoff for patient grouping}

In many cases, gene expression follows a bi-modal distribution with unbalanced sample sizes in each group. A cluster-based cutoff selection is provided to assist identification of an optimal cutoff value for group dichotomization in survival analysis. $\mathrm{R}$ package mclust [46] was used to identify the gene expression cutoff based on Gaussian mixture model clustering, assuming a bi-modal distribution when users select the "cluster" option under the survival analysis module of LCE.

\section{Survival analysis}

Survival curves were estimated using the product-limit method of Kaplan-Meier [47] (survival, R package [48]). A log-rank test was used to compare the survival differences among different patient groups. A Cox proportional hazard regression model was used to assess the survival association and calculate the hazard ratio (HR) with continuous gene expression in each individual dataset.

\section{Meta-analysis}

For survival meta-analysis, the $\mathrm{R}$ package meta [49] was used to calculate the summary HR from the HRs of individual datasets. For tumor vs normal differential expression meta-analysis, R package metafor [50] was used to calculate the summary standardized mean difference (tumor - normal) using Hedges' $G$ as an effect size metric.

\section{Comparative analysis}

For comparative analysis, Welch's two-sample t-test assuming unequal variance was used to generate the $p$ value. In the resulting box whisker plot, the lower whisker extends from the lower quartile to the lowest smaller value within 1.5 inter-quartile-range (IQR), whereas the upper whisker extends from the upper quartile to the highest larger value within 1.5 IQR. The red solid dot and the value beside it represent the group mean.

\section{Correlation analysis}

For correlation analysis, the Pearson correlation was used to calculate the correlation coefficients. The dendrogram for the heatmap was generated based on complete-linkage hierarchical clustering of the correlation coefficients based on Euclidean distance.

\section{Data availability}

All the datasets were downloaded from the public domain. The processed and normalized data are available upon request. The web-portal we developed in this study can be accessed through the following link: http://lce.biohpc.sw med.edu/

Acknowledgements This work was partially supported by grants from the National Institutes of Health [5R01CA152301, P50CA70907, 5P30CA142543, 1R01GM115473, and 1R01CA172211], and the Cancer Prevention and Research Institute of Texas [RP120732, RP180805, and RP150596]. We thank Jessie Norris for proofreading the manuscript.

Author contributions YX, JM, and GX supervised the project. LC, YX, and GX conceived the method. LC designed and performed the analyses and interpreted the results. LC, YZ, LY, and BC curated the data. With advice from GX, JM, and YX, SL, LC, BC, QZ, DL, JA, and $\mathrm{BY}$ developed the web application. $\mathrm{LY}, \mathrm{KH}, \mathrm{AG}, \mathrm{JH}$, and IW provided critical input. LC drafted the article. GX, YX, and JM critically edited the article. All co-authors have read and edited the manuscript.

\section{Compliance with ethical standards}

Conflict of interest The authors declare that they have no conflict of interest.

Open Access This article is licensed under a Creative Commons Attribution 4.0 International License, which permits use, sharing, adaptation, distribution and reproduction in any medium or format, as long as you give appropriate credit to the original author(s) and the source, provide a link to the Creative Commons license, and indicate if changes were made. The images or other third party material in this article are included in the article's Creative Commons license, unless indicated otherwise in a credit line to the material. If material is not included in the article's Creative Commons license and your intended use is not permitted by statutory regulation or exceeds the permitted use, you will need to obtain permission directly from the copyright holder. To view a copy of this license, visit http://creativecommons. org/licenses/by/4.0/.

\section{References}

1. Siegel RL, Miller KD, Jemal A. Cancer statistics, 2018. CA Cancer J Clin. 2018;68:7-30.

2. Kris MG, Johnson BE, Berry LD, Kwiatkowski DJ, Iafrate AJ, Wistuba II, et al. Using multiplexed assays of oncogenic drivers in lung cancers to select targeted drugs. JAMA. 2014;311:19982006.

3. Zappasodi R, Merghoub T, Wolchok JD. Emerging concepts for immune checkpoint blockade-based combination therapies. Cancer Cell. 2018;33:581-98.

4. Thorsson V, Gibbs DL, Brown SD, Wolf D, Bortone DS, Ou Yang $\mathrm{TH}$, et al. The immune landscape of cancer. Immunity. 2018;48:812-30. e14

5. Rizvi H, Sanchez-Vega F, La K, Chatila W, Jonsson P, Halpenny $\mathrm{D}$, et al. Molecular determinants of response to anti-programmed cell death (PD)-1 and anti-programmed death-ligand 1 (PD-L1) 
blockade in patients with non-small-cell lung cancer profiled with targeted next-generation sequencing. J Clin Oncol. 2018;36:63341.

6. Hellmann MD, Ciuleanu TE, Pluzanski A, Lee JS, Otterson GA, Audigier-Valette $\mathrm{C}$, et al. Nivolumab plus ipilimumab in lung cancer with a high tumor mutational burden. $\mathrm{N}$ Engl $\mathrm{J}$ Med. 2018;378:2093-104.

7. Hellmann MD, Callahan MK, Awad MM, Calvo E, Ascierto PA, Atmaca A, et al. Tumor mutational burden and efficacy of nivolumab monotherapy and in combination with ipilimumab in smallcell lung cancer. Cancer Cell. 2018;33:853-61. e4

8. Edgar R, Domrachev M, Lash AE. Gene Expression Omnibus: NCBI gene expression and hybridization array data repository. Nucleic Acids Res. 2002;30:207-10.

9. Parkinson H, Sarkans U, Shojatalab M, Abeygunawardena N, Contrino S, Coulson R, et al. ArrayExpress-a public repository for microarray gene expression data at the EBI. Nucleic Acids Res. 2005;33(Database issue):D553-5.

10. Brazma A, Parkinson H, Sarkans U, Shojatalab M, Vilo J, Abeygunawardena N, et al. ArrayExpress-a public repository for microarray gene expression data at the EBI. Nucleic Acids Res. 2003;31:68-71.

11. Rhodes DR, Kalyana-Sundaram S, Mahavisno V, Varambally R, Yu J, Briggs BB, et al. Oncomine 3.0: genes, pathways, and networks in a collection of 18,000 cancer gene expression profiles. Neoplasia. 2007;9:166-80.

12. Kilpinen S, Autio R, Ojala K, Iljin K, Bucher E, Sara H, et al. Systematic bioinformatic analysis of expression levels of 17,330 human genes across 9,783 samples from 175 types of healthy and pathological tissues. Genome Biol. 2008;9:R139.

13. Zoubarev A, Hamer KM, Keshav KD, McCarthy EL, Santos JR, Van Rossum T, et al. Gemma: a resource for the reuse, sharing and meta-analysis of expression profiling data. Bioinformatics. 2012;28:2272-3.

14. Cheng WC, Tsai ML, Chang CW, Huang CL, Chen CR, Shu WY, et al. Microarray meta-analysis database (M(2)DB): a uniformly pre-processed, quality controlled, and manually curated human clinical microarray database. BMC Bioinforma. 2010;11:421.

15. Feichtinger J, McFarlane RJ, Larcombe LD. CancerMA: a webbased tool for automatic meta-analysis of public cancer microarray data. Database. 2012;2012:bas055.

16. Gao J, Aksoy BA, Dogrusoz U, Dresdner G, Gross B, Sumer SO, et al. Integrative analysis of complex cancer genomics and clinical profiles using the cBioPortal. Sci Signal. 2013;6:pl1.

17. Szasz AM, Lanczky A, Nagy A, Forster S, Hark K, Green JE, et al. Cross-validation of survival associated biomarkers in gastric cancer using transcriptomic data of 1,065 patients. Oncotarget. 2016;7:49322-33.

18. Mizuno H, Kitada K, Nakai K, Sarai A. PrognoScan: a new database for meta-analysis of the prognostic value of genes. BMC Med Genom. 2009;2:18.

19. Goswami CP, Nakshatri H. PROGgene: gene expression based survival analysis web application for multiple cancers. J Clin Bioinform. 2013;3:22.

20. Orvis T, Hepperla A, Walter V, Song S, Simon J, Parker J, et al. BRG1/SMARCA4 inactivation promotes non-small cell lung cancer aggressiveness by altering chromatin organization. Cancer Res. 2014;74:6486-98.

21. Fukuoka J, Fujii T, Shih JH, Dracheva T, Meerzaman D, Player A, et al. Chromatin remodeling factors and BRM/BRG1 expression as prognostic indicators in non-small cell lung cancer. Clin Cancer Res. 2004;10:4314-24.

22. Bai J, Mei P, Zhang C, Chen F, Li C, Pan Z, et al. BRG1 is a prognostic marker and potential therapeutic target in human breast cancer. PLoS ONE. 2013;8:e59772.
23. Director's Challenge Consortium for the Molecular Classification of Lung A, Shedden K, Taylor JM, Enkemann SA, Tsao MS, Yeatman TJ, et al. Gene expression-based survival prediction in lung adenocarcinoma: a multi-site, blinded validation study. Nat Med. 2008;14:822-7.

24. Schabath MB, Welsh EA, Fulp WJ, Chen L, Teer JK, Thompson ZJ, et al. Differential association of STK11 and TP53 with KRAS mutation-associated gene expression, proliferation and immune surveillance in lung adenocarcinoma. Oncogene. 2016;35:320916.

25. Torres PU, Prie D, Molina-Bletry V, Beck L, Silve C, Friedlander G. Klotho: an antiaging protein involved in mineral and vitamin D metabolism. Kidney Int. 2007;71:730-7.

26. Kurosu H, Yamamoto M, Clark JD, Pastor JV, Nandi A, Gurnani $\mathrm{P}$, et al. Suppression of aging in mice by the hormone Klotho. Science. 2005;309:1829-33.

27. Morishita K, Shirai A, Kubota M, Katakura Y, Nabeshima Y, Takeshige $\mathrm{K}$, et al. The progression of aging in klotho mutant mice can be modified by dietary phosphorus and zinc. J Nutr. 2001;131:3182-8.

28. Xie B, Chen J, Liu B, Zhan J. Klotho acts as a tumor suppressor in cancers. Pathol Oncol Res. 2013;19:611-7.

29. Zhang A, Wang C, Wang S, Li L, Liu Z, Tian S. Visualizationaided classification ensembles discriminate lung adenocarcinoma and squamous cell carcinoma samples using their gene expression profiles. PLoS ONE. 2014;9:e110052.

30. Higgins JP, Thompson SG. Quantifying heterogeneity in a metaanalysis. Stat Med. 2002;21:1539-58.

31. Nordenberg D, Yip R, Binkin NJ. The effect of cigarette smoking on hemoglobin levels and anemia screening. JAMA. 1990;264:1556-9.

32. Niehrs C, Pollet N. Synexpression groups in eukaryotes. Nature. 1999;402:483-7.

33. Greene CS, Krishnan A, Wong AK, Ricciotti E, Zelaya RA, Himmelstein DS, et al. Understanding multicellular function and disease with human tissue-specific networks. Nat Genet. 2015;47:569-76.

34. Fabregat A, Jupe S, Matthews L, Sidiropoulos K, Gillespie M, Garapati P, et al. The Reactome Pathway Knowledge base. Nucleic Acids Res. 2018;46:D649-55.

35. Liberzon A, Subramanian A, Pinchback R, Thorvaldsdottir H, Tamayo P, Mesirov JP. Molecular signatures database (MSigDB) 3.0. Bioinformatics. 2011;27:1739-40.

36. Ame JC, Rolli V, Schreiber V, Niedergang C, Apiou F, Decker P, et al. PARP-2, A novel mammalian DNA damage-dependent poly (ADP-ribose) polymerase. J Biol Chem. 1999;274:17860-8.

37. Gray KA, Yates B, Seal RL, Wright MW, Bruford EA. Genenames.org: the HGNC resources in 2015. Nucleic Acids Res. 2015;43(Database issue):D1079-85.

38. Zhu Y, Davis S, Stephens R, Meltzer PS, Chen Y. GEOmetadb: powerful alternative search engine for the Gene Expression Omnibus. Bioinformatics. 2008;24:2798-800.

39. Omberg L, Ellrott K, Yuan Y, Kandoth C, Wong C, Kellen MR, et al. Enabling transparent and collaborative computational analysis of 12 tumor types within The Cancer Genome Atlas. Nat Genet. 2013;45:1121-6.

40. Travis WD, Brambilla E, Nicholson AG, Yatabe Y, Austin JH, Beasley MB, et al. The 2015 World Health Organization classification of lung tumors: impact of genetic, clinical and radiologic advances since the 2004 classification. J Thorac Oncol. 2015;10:1243-60.

41. Andrew G Nicholson, Keith Kerr, John Gosney. G048 Dataset for histopathological reporting of lung cancer. The Royal College of Pathologists. 2018.

42. Girard L, Rodriguez-Canales J, Behrens C, Thompson DM, Botros IW, Tang H, et al. An expression signature as an aid to the 
histologic classification of non-small cell lung cancer. Clin Cancer Res. 2016;22:4880-9.

43. Broad Institute TCGA Genome Data Analysis Center (2016): Analysis-ready standardized TCGA data from Broad GDAC Firehose 2016_01_28 run. Broad Institute of MIT and Harvard. Dataset. https://doi.org/10.7908/C11G0KM9

44. Parmigiani G, Garrett-Mayer ES, Anbazhagan R, Gabrielson E. A cross-study comparison of gene expression studies for the molecular classification of lung cancer. Clin Cancer Res. 2004;10:2922-7.

45. Kang DD, Sibille E, Kaminski N, Tseng GC. MetaQC: objective quality control and inclusion/exclusion criteria for genomic metaanalysis. Nucleic Acids Res. 2012;40:e15.
46. Chris Fraley, Adrian E. Raftery. Model-based clustering, discriminant analysis and density estimation. J Am Stat Assoc. 2002;97:611-31.

47. Kaplan EL, Meier P. Nonparametric estimation from incomplete observations. J Am Stat Assoc; 1958;53:457-81.

48. Therneau, Terry M., Grambsch, Patricia M. Modeling survival data: extending the Cox model: Springer; 2000.

49. Guido Schwarzer. meta: An R package for meta-analysis. R News. 2007;7:40-5.

50. Viechtbauer W. Conducting meta-analyses in $\mathrm{R}$ with the metafor package. J Stat Softw. 2010;36:1-48. 